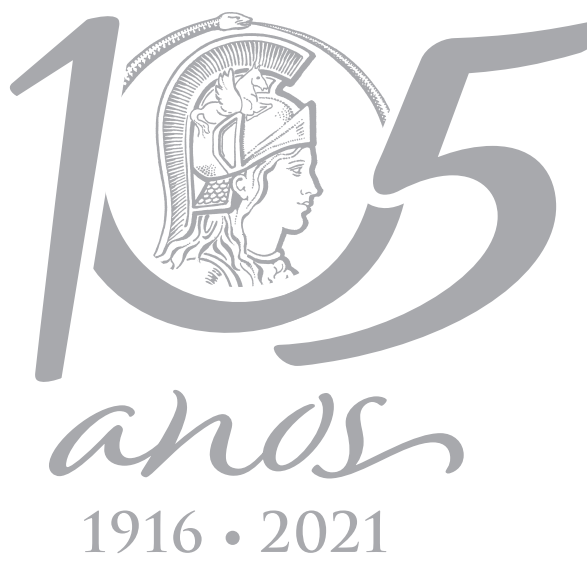

An Acad Bras Cienc (2021) 93(4): e20191139 DOI 10.1590/0001-3765202120191139

Anais da Academia Brasileira de Ciências | Annals of the Brazilian Academy of Sciences

Printed ISSN 0001-3765 I Online ISSN 1678-2690

www.scielo.br/aabc | www.fb.com/aabcjournal

MATHEMATICAL SCIENCES

\title{
Rational first integrals of the Liénard equations: The solution to the Poincaré problem for the Liénard equations
}

\author{
JAUME LLIBRE, CLAUDIO PESSOA \& JARNE D. RIBEIRO
}

\begin{abstract}
Poincaré in 1891 asked about the necessary and sufficient conditions in order to characterize when a polynomial differential system in the plane has a rational first integral. Here we solve this question for the class of Liénard differential equations $\ddot{x}+$ $f(x) \dot{x}+x=0$, being $f(x)$ a polynomial of arbitrary degree. As far as we know it is the first time that all rational first integrals of a relevant class of polynomial differential equations of arbitrary degree has been classified.
\end{abstract}

Key words: Liénard equation, rational first integral, Poincaré problem, polinomial differential equation.

\section{1 - THE POINCARÉ PROBLEM ON THE RATIONAL FIRST INTEGRALS OF THE POLYNOMIAL DIFFERENTIAL SYSTEMS}

A rational function $f(x, y) / g(x, y)$ has degree $m$ if the polynomials $f(x, y)$ and $g(x, y)$ are coprime in the ring $\mathbb{R}[x, y]$, and the maximum of the degrees of $f(x, y)$ and $g(x, y)$ is $m$.

A polynomial differential system is a differential system of the form

$$
\frac{d x}{d t}=\dot{x}=P(x, y), \quad \frac{d y}{d t}=\dot{x}=Q(x, y),
$$

where $P(x, y)$ and $Q(x, y)$ are real polynomials in the variables $x$ and $y$, and $t$ is the independent variable usually called the time. The polynomial vector field associated to the polynomial differential system (1) is

$$
\mathcal{X}=P(x, y) \frac{\partial}{\partial x}+Q(x, y) \frac{\partial}{\partial y} .
$$

Let $U$ be an open subset of $\mathbb{R}^{2}$. Here a first integral is a $\mathcal{C}^{1}$ non-locally constant function $H: U \rightarrow \mathbb{R}$ such that it is constant on the solutions $(x(t), y(t))$ of the polynomial differential system (1) contained in $U$, i.e. if $\left.\mathcal{X}(H)\right|_{U} \equiv 0$.

If the function $H$ is rational then we say that $H$ is a rational first integral.

The problem of providing necessary and sufficient conditions in order that a polynomial differential system in the plane has a rational first integral was stated by Poincaré (1891). This problem 
is of a global nature involving whole classes of polynomial differential systems and this is one of the reasons for being so hard.

If $\mathcal{X}$ is a polynomial vector field on $\mathbb{R}^{2}$ the $n$-th extactic curve of $\mathcal{X}, \mathcal{E}_{n}(\mathcal{X})$, is defined by the polynomial equation

$$
\operatorname{det}\left(\begin{array}{cccc}
v_{1} & v_{2} & \cdots & v_{l} \\
\mathcal{X}\left(v_{1}\right) & \mathcal{X}\left(v_{2}\right) & \cdots & \mathcal{X}\left(v_{l}\right) \\
\vdots & \vdots & \cdots & \vdots \\
\mathcal{X}^{l-1}\left(v_{1}\right) & \mathcal{X}^{l-1}\left(v_{2}\right) & \cdots & \mathcal{X}^{l-1}\left(v_{l}\right)
\end{array}\right)=0
$$

where $v_{1}, v_{2}, \cdots, v_{l}$ is a basis of $\mathbb{R}_{n}[x, y]$, the $\mathbb{R}$-vector space formed by all polynomials in $\mathbb{R}[x, y]$ of degree at most $n$, and so $l=(n+1)(n+2) / 2$, and $\mathcal{X}^{j}\left(v_{i}\right)=\mathcal{X}^{j-1}\left(\mathcal{X}\left(v_{i}\right)\right)$. Observe that the definition of extactic curve is independent of the chosen basis of the $\mathbb{R}$-vector space of polynomials of degree at most $n$, and that the extactic curve is an algebraic curve. See Christopher et al. (2007) for more details about the extactic curve.

As far as we know the first solution for the problem mentioned above was given in the next result.

Theorem 1. Let $\mathcal{X}$ be a polynomial vector field. Then the polynomial $\mathcal{E}_{n}(\mathcal{X})$ is identically zero and the polynomial $\mathcal{E}_{n-1}(\mathcal{X})$ is not identically zero if, and only if, $\mathcal{X}$ admits a rational first integral of degree $n>1$.

This result is Theorem 4.3 of the paper Christopher et al. (2007). But in general Theorem 1 is difficult to apply because if the degree of the rational first integral is higher, then the computation of the determinant which appears in the definition of $\mathcal{E}_{n}(\mathcal{X})$ is not easy.

\section{2 - THE SOLUTION TO THE POINCARÉ PROBLEM FOR THE LIÉNARD EQUATIONS}

One of the most studied classes of polynomial differential equations is the Liénard differential equations, or simply Liénard equations

$$
\ddot{x}+f(x) \dot{x}+x=0,
$$

where $f(x)$ is a polynomial. The first in considering the differential equations of the form (2) was Liénard (1928) during the development of radio and vacuum tube technology. Later on these equations were intensely studied as they can be used to model oscillating circuits, see for instance the classical books Andronov et al. (1987), Conti et al. (1969), Lefschetz (1957) and Sansone (1948).

Passing to the Liénard plane the second order differential equation (2) is equivalent to the first order polynomial differential system

$$
\dot{x}=y-F(x), \quad \dot{y}=-x,
$$

where $F(x)=\int_{0}^{x} f(s) d s$.

Another way to write the second order differential equation (2) as a planar differential system of first order is

$$
\dot{x}=y, \quad \dot{y}=-f(x) y-x .
$$


The objective of this paper is to solve the problem stated by Poincare on the existence of rational first integrals for the class of polynomial Liénard differential systems (3), and consequently also for the equivalent classes of differential equations (2) and (4).

Consider the polynomial differential systems (3) in $\mathbb{R}^{2}$ where $F(x)=F_{n}(x)$ is polynomial in $x$ of degree $n \geq 1$. These differential systems are called simply Liénard systems.

We denote by

$$
\mathcal{X}=\left(y-F_{n}(x)\right) \frac{\partial}{\partial x}-x \frac{\partial}{\partial y}
$$

the polynomial vector field associated to system (3).

The Totiente Euler function $\varphi(x)$ is such that for each $x \in \mathbb{N}=\{1,2,3, \ldots\}, \varphi(x)$ is the quantity of numbers $k \in\{1,2, \ldots, x\}$ such that $(k, x)=1$, that is $x$ and $k$ are relatively prime.

$$
\varphi(x)=\#\{n \in \mathbb{N}: n \leq x \wedge(n, x)=1\} .
$$

The fundamental theorem of arithmetic states that if $x>1$ there is a unique expression for $x=$ $p_{1}^{k_{1}} p_{2}^{k_{2}} \cdots p_{r}^{k_{r}}$, where $1<p_{1}<p_{2}<\cdots<p_{r}$ are prime numbers and each integer $k_{i} \geqslant 1$. Then the function $\varphi(x)$ has following expression

$$
\varphi(x)=x \prod_{p \mid x}\left(1-\frac{1}{p}\right),
$$

where the product is over the distinct prime numbers dividing $x$, for more details on the Totiente Euler function see Theorem 62 of Hardy \& Wright (1979).

Our main results are the following two theorems.

Theorem 2. For all $m \geq 1$ different from 2 there are $2 \varphi(m)$ Liénard systems (3) of degree 1 with a rational first integral of degree $m$, and for $m=2$ there are $2 \varphi(m)+1$ Liénard systems (3) of degree 1 with a rational first integral of degree 2 .

Theorem 3. There are no Liénard systems (3) of degree > 1 having rational first integrals.

We proved Theorems 2 and 3 in the next section.

We note that Theorems 2 and 3 characterize all the Liénard equations (2) which have rational first integrals. As far as we know it is the first time that all rational first integrals of a relevant class of polynomial differential equations of arbitrary degree has been classified.

We remark that the limit cycles of the Liénard equations (2) has been intensively studied, see for instance Dumortier et al. (2007), Dumortier \& Maesschalck (2011), Hirsch et al. (1977), Huzak \& Maesschalck (2015), Li \& Llibre (2012) and Llibre \& Zang (2017). But it remains many open questions about these limit cycles.

\section{3 - PROOF OF THE RESULTS}

Proof of Theorem 2. We consider the Liénard system of degree 1 given by

$$
\dot{x}=y-a_{0}-a_{1} x, \quad \dot{y}=-x .
$$


It is easy to check that system (5) is integrable with the first integral

$$
\begin{aligned}
H= & \left(a_{1}\left(\sqrt{\frac{a_{1}^{2}-4}{a_{1}^{2}}}-1\right)\left(a_{0}+a_{1} x-y\right)+2 x\right)^{1-\frac{a_{1}^{2}}{2}}\left(\sqrt{1-\frac{4}{a_{1}^{2}}}+1\right) \\
& \left(a_{1}\left(\sqrt{\frac{a_{1}^{2}-4}{a_{1}^{2}}}+1\right)\left(a_{0}+a_{1} x-y\right)-2 x\right)
\end{aligned}
$$

So in order that from the expression of the function $\mathrm{H}$ we can obtain rational first integrals of degree $m=1$ we must have

$$
1-\frac{a_{1}^{2}}{2}\left(\sqrt{1-\frac{4}{a_{1}^{2}}}+1\right)=-1,
$$

i.e. $a_{1}= \pm 2$. This proves the theorem for $m=1$.

Again from the expression of the function $H$ we can obtain rational first integrals of degree $m>1$ we must have

$$
1-\frac{a_{1}^{2}}{2}\left(\sqrt{1-\frac{4}{a_{1}^{2}}}+1\right)=-\frac{m}{r},
$$

with $r \in\{1, \ldots, m-1\}$ and $(r, m)=1$. Solving equations ( 7$)$ with respect to $a_{1}$ we get

$$
a_{1}= \pm \frac{m+r}{\sqrt{m r}}
$$

So, for a given positive integer $m>1$ we have $\varphi(m)$ good numbers $m / r$ for which from the expression of $H$ we can obtain $\varphi(m)$ different Liénard systems of degree 1 with a rational first integral of degree $m$. This proves the theorem for $m>2$.

For $m=2$ we note that if $a_{1}=0$ then system (5) is Hamiltonian with the first integral $H=$ $\left(x^{2}+\left(y-a_{0}\right)^{2}\right) / 2$. So in this case the number of rational first integrals is $2 \varphi(2)+1$. This completes the proof of the theorem.

From Theorem 2 we provide the explicit Liénard systems with a rational first integral $H$ of degree 2,3 and 4 .

Example 4. Consider $m=2$. From Theorem 2 there are two Liénard systems of degree 1 with $\mathrm{H}$ a rational first integral of degree 2 , which are given by:

For $r=1$,

$$
\begin{gathered}
\dot{x}=y-a_{0}+3 x / \sqrt{2}, \quad \dot{y}=-x, \quad \text { with } \quad H=\frac{-\sqrt{2} a_{0}+2 x+\sqrt{2} y}{\left(-\sqrt{2} a_{0}+x+\sqrt{2} y\right)^{2}}, \\
\dot{x}=y-a_{0}-3 x / \sqrt{2}, \quad \dot{y}=-x, \quad \text { with } \quad H=\frac{\sqrt{2} a_{0}+2 x-\sqrt{2} y}{\left(\sqrt{2} a_{0}+x-\sqrt{2} y\right)^{2}} .
\end{gathered}
$$

Example 5. Consider $m=3$. From Theorem 2 there are four Liénard systems of degree 1 with $\mathrm{H} \mathrm{a}$ rational first integral of degree 3 , which are given by: 
For $r=1$,

$$
\begin{gathered}
\dot{x}=y-a_{0}+4 x / \sqrt{3}, \quad \dot{y}=-x, \quad \text { with } \quad H=-\frac{-\sqrt{3} a_{0}+3 x+\sqrt{3} y}{\left(-\sqrt{3} a_{0}+x+\sqrt{3} y\right)^{3}}, \\
\dot{x}=y-a_{0}-4 x / \sqrt{3}, \quad \dot{y}=-x, \quad \text { with } \quad H=-\frac{\sqrt{3} a_{0}+3 x-\sqrt{3} y}{\left(\sqrt{3} a_{0}+x-\sqrt{3} y\right)^{3}} .
\end{gathered}
$$

For $r=2$,

$$
\begin{aligned}
& \dot{x}=y-a_{0}+5 x / \sqrt{6}, \quad \dot{y}=-x, \quad \text { with } H=\frac{\left(-2 a_{0}+\sqrt{6} x+2 y\right)^{2}}{\left(\sqrt{6} a_{0}-2 x-\sqrt{6} y\right)^{3}}, \\
& \dot{x}=y-a_{0}-5 x / \sqrt{6}, \quad \dot{y}=-x, \quad \text { with } \quad H=\frac{\left(2 a_{0}+\sqrt{6} x-2 y\right)^{2}}{\left(-\sqrt{6} a_{0}-2 x+\sqrt{6} y\right)^{3}} .
\end{aligned}
$$

Example 6. Consider $m=4$. From Theorem 2 there are four Liénard systems of degree 1 with $\mathrm{H} \mathrm{a}$ rational first integral of degree 4 , which are given by:

For $r=1$,

$$
\begin{gathered}
\dot{x}=y-a_{0}+5 x / 2, \quad \dot{y}=-x, \quad \text { with } \quad H=\frac{-a_{0}+2 x+y}{\left(-2 a_{0}+x+2 y\right)^{4}}, \\
\dot{x}=y-a_{0}-5 x / 2, \quad \dot{y}=-x, \quad \text { with } \quad H=\frac{a_{0}+2 x-y}{\left(2 a_{0}+x-2 y\right)^{4}} .
\end{gathered}
$$

For $r=3$,

$$
\begin{aligned}
& \dot{x}=y-a_{0}+7 x / \sqrt{12}, \quad \dot{y}=-x, \quad \text { with } \quad H=\frac{\left(-\sqrt{3} a_{0}+2 x+\sqrt{3} y\right)^{3}}{\left(2 \sqrt{3} a_{0}-3 x-2 \sqrt{3} y\right)^{4}} \\
& \dot{x}=y-a_{0}-7 x / \sqrt{12}, \quad \dot{y}=-x, \quad \text { with } \quad H=\frac{\left(\sqrt{3} a_{0}+2 x-\sqrt{3} y\right)^{3}}{\left(-2 \sqrt{3} a_{0}-3 x+2 \sqrt{3} y\right)^{4}} .
\end{aligned}
$$

Let $f$ be a real polynomial in the variables $x$ and $y$. The algebraic curve $f=f(x, y)=0$ is an invariant algebraic curve of a polynomial differential system (1) if for some polynomial $K=K(x, y)$ we have

$$
\mathcal{X} f=P \frac{\partial f}{\partial x}+Q \frac{\partial f}{\partial y}=K f
$$

Since on the points of the algebraic curve $f=0$ the gradient $(\partial f / \partial x, \partial f / \partial y$ ) of the curve $f(x, y)=0$ is orthogonal to the vector field $\mathcal{X}=(P, Q)$ (see (8)), on the points of $f=0$ the vector field $\mathcal{X}$ is tangent to the curve $f=0$. Therefore the curve $f=0$ is formed by trajectories of the vector field $\mathcal{X}$. This justifies the name of invariant algebraic curve given to the algebraic curve $f=0$ satisfying (8) for some polynomial $K$, because it is invariant under the flow defined by $\mathcal{X}$. 
Darboux (1878) found for polynomial differential systems a fascinating relationships between the integrability (a topological phenomena) and the existence of a sufficient number of invariant algebraic solutions. In particular, he proved that if a polynomial differential system of degree $m$ has at least $m(m+1) / 2$ invariant algebraic curves, then it has a first integral. Moreover, if the number of invariant algebraic curves is at least $m(m+1) / 2+2$ then there is a first integral which is a rational function, see for instance Christopher \& Llibre (2000).

To prove Theorem 3 we use the following result. Hayashi (1996) studied the invariant algebraic curves for the Liénard system

$$
\dot{x}=y, \quad \dot{y}=-f(x) y-g(x),
$$

where $f$ and $g$ are polynomials of degree $M$ and $N$ respectively and obtained the following result.

Theorem 7. Under the conditions $f(x) \neq 0$, and $M+1 \geq N$ the Liénard system (9) has an invariant algebraic curve if and only if there is an invariant curve $y=P(x)$ satisfying

$$
g(x)=-\left[f(x)+P^{\prime}(x)\right] P(x),
$$

where $P(x)$ or $P(x)+\int f(x) d x$ is a polynomial of degree at most one.

Proof of Theorem 3. Consider system (3) of degree $n>1$, with $F(x)=\sum_{i=0}^{n} a_{i} x^{i}$ and $a_{n} \neq 0$.

System (3) is equivalent to system (9) if $f(x)=F^{\prime}(x)$ and $g(x)=x$. Therefore by Theorem 7 system

(3) has an invariant algebraic curve if and only if

(i) $P(x)=d_{0}+d_{1} x$, and

$$
g(x)=-\left[f(x)+P^{\prime}(x)\right] P(x) \Leftrightarrow x=-\left[f(x)+d_{1}\right]\left(d_{0}+d_{1} x\right) .
$$

Then from last equation we obtain that $-n a_{n} d_{1} x^{n}=0$, a contradiction if $d_{1} \neq 0$. If $d_{1}=0$ then get $-n a_{n} d_{0} x^{n-1}=0$, a contradiction $d_{0} \neq 0$. If $d_{1}=d_{0}=0$ then we obtain a contradiction in the last equation of (10).

(ii) $P(x)=d_{0}+d_{1} x-F(x)$. Since the degree of $F(x)$ is larger than one, this condition never holds. Therefore system (3) with $n>1$ has no invariant algebraic curves, so systems (3) cannot have rational first integrals.

\section{Acknowledgments}

The first author is partially supported by the Ministerio de Ciencia, Innovación y Universidades, Agencia Estatal de Investigación grants MTM2016-77278-P (FEDER), the Agència de Gestió d'Ajuts Universitaris i de Recerca grant 2017SGR1617, and the H2O20 European Research Council grant MSCA-RISE-2017-777911.

The second author is partially supported by Coordenação de Aperfeiçoamento de Pessoal de Nível Superior/ Programa Nacional de Cooperação Acadêmica (CAPES PROCAD) grant 88881.068462/2014-01 and by Fundação de Amparo à Pesquisa do Estado de São Paulo (FAPESP) grants 18/19726-5 and 19/10269-3.

The third author is partially supported by the Instituto Federal de Educação, Ciência e Tecnologia do Sul de Minas Gerais - IFSULDEMINAS. 


\section{REFERENCES}

ANDRONOV AA, KHAIKIN SE \& VITT AA. 1987. Theory of oscillators. Dover, reprint, $815 \mathrm{p}$.

CHRISTOPHER C \& LLIBRE J. 2000. Integrability via invariant algebraic curves for planar polynomial differential systems. Ann Differential Equations 16: 5-19.

CHRISTOPHER C, LLIBRE J \& PEREIRA JV. 2007. Multiplicity of invariant algebraic curves in polynomial vector fields. Pacific J of Math 229: 63-117.

CONTI R, REISSIG R \& SANSONE G. 1969. Nichtlineare Differentialgleichungen höherer Ordnung. Edizioni Cremonese, $738 \mathrm{p}$.

DARBOUX G. 1878. Mémoire sur les équations différentielles algébriques du premier ordre et du premier degré. Bull Sci Math Ast 2e, série 2, 1: 60-96.

DUMORTIER F, PANAZZOLO D \& ROUSSARIE R. 2007. More limit cycles than expected in Liénard equations. Proc Amer Math Soc 135: 1895-1904.

DUMORTIER F \& MAESSCHALCK PD. 2011. Classical Liénard equation of degree $n \geq 6$ can have $\left[\frac{n-1}{2}\right]+2$ limit cycles. J Differ Equ 250: 2162-2176.

HARDY GH \& WRIGHT EM. 1979. An Introduction to the Theory of Numbers. Oxford University Press, $426 \mathrm{p}$.

HAYASHI M. 1996. On polynomial Liénard systems which have invariant algebraic curves. Funkcial Ekvac 39: 403-408.

HIRSCH M, PUGH C \& SHUB M. 1977. Invariant Manifolds. Lecture Notes in Math 583, Springer-Verlag, 149 p.

HUZAK R \& MAESSCHALCK PD. 2015. Slow divergence integrals in classical Liénard equations near centers. J Dyn Diff Equat 27: 117-185.

LEFSCHETZ S. 1957. Differential equations: geometric theory. Pure and Applied Mathematics, Vol. VI, Interscience Publishers, $364 \mathrm{p}$.

LI C \& LLIBRE J. 2012. Uniqueness of limit cycle for Liénard equations of degree four. J Differ Equ 252: 3142-3162.

LIÉNARD A. 1928. Etude des oscillations entretenues, Rev Gen de l'Electricite 23: 901-912/946-954.

LLIBRE J \& ZANG X. 2017. Limit cycles of the classical Liénard differential systems: A survey on the Lins Neto, de Melo and Pugh's conjecture. Expo Math 35: 286-299.

POINCARÉ H. 1891. Sur l'intégration algébrique des équations différentielles du premier ordre et du premier degré I and II. Rendiconti del Circolo Matematico di Palermo Vol. 5, p. 161-191.
SANSONE G. 1948. Ordinary differential equations. Zanichelli 1948, $400 \mathrm{p}$.

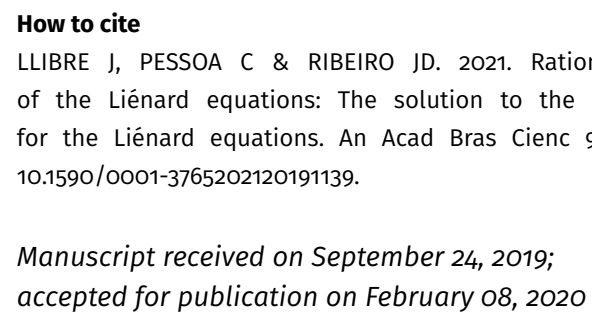

Manuscript received on September 24, 2019;

accepted for publication on February 08, 2020

\section{JAUME LLIBRE}

https://orcid.org/0000-0002-9511-5999

\section{CLAUDIO PESSOA ${ }^{2}$}

https://orcid.org/0000-0001-6790-1055

JARNE D. RIBEIRO 3

https://orcid.org/0000-0001-9729-900X

${ }^{1}$ Departament de Matemàtiques, Universitat Autònoma de Barcelona, 08193 Bellaterra, Barcelona, Catalonia, Spain

${ }^{2}$ Departamento de Matemática, Universidade Estadual Paulista, Campus São José do Rio Preto, IBILCE, R. Cristóvão Colombo 2265, 15054-000 São José do Rio Preto, SP, Brazil

3 Instituto Federal de Educação, Ciência e Tecnologia do Sul de Minas Gerais, IFSULDEMINAS, Rua Mario Ribola 409, Penha II, 37903-358 Passos, MG, Brazil

Correspondence to: Claudio G. Pessoa

E-mail: c.pessoa@unesp.br

\section{Author contributions}

Both authors worked on the mathematical solution of the studied problem. Furthermore, Jaume Llibre took the initiative to propose the study of the problem, Jarne D. Ribeiro wrote the original draft and Claudio Pessoa did the general revision and corrections of the paper.

\section{(cc) BY}

